\title{
Quantitative Measurement of Respiratory Split in the Second Heart Sound
}

\author{
Hong Tang ${ }^{1}$, Huaming Chen ${ }^{1}$, Ting $\mathrm{Li}^{2}$ \\ ${ }^{1}$ Dalian University of Technology, Dalian, China \\ ${ }^{2}$ Dalian Minzu University, Dalian, China
}

\begin{abstract}
The second heart sound (S2) consists of aortic and pulmonary components. Physiological findings disclose that the split between the two components is modulated by respiration. It is assumed that the waveforms of the aortic and pulmonary components remain constant in adjective cardiac cycles. Then, the S2s are aligned with respect to the aortic component in time domain. So, the average of the aligned S2s produces the aortic component, and the pulmonary component tends to disappear due to the average operation. The average is taken as the template of the aortic component. The pulmonary component of a cardiac cycle is separated by subtracting the template from the S2 of this cycle. The timing of a component is defined as the weighted energy peak locations. Therefore, the split between the two components is measured by the timing difference. Data collected from 12 healthy human subjects are used to evaluate the method. The experiment results show that the minimum split occurs at the end of expiration and its value is about $20 \mathrm{~ms}$. Meanwhile, the maximum split is about $50 \mathrm{~ms}$ at the end of inspiration. Both the trend of split varying with respect to respiratory phase and the numerical range of split varying are comparable to the previous results. The timing difference measures successfully the split modulated by respiration. This method has potential applications in monitoring hemodynamic response to respiration.
\end{abstract}

\section{Introduction}

Heart sounds are mechanical vibrations generated by beating heart and blood flow therein [1-2]. Normal heart sounds in a cardiac cycle, in general, consist of the first heart sounds (S1) and the second heart sounds (S2). Previous studies disclosed that the first component of S1 followed the onset of the left ventricular pressure rise and closure of the mitral valve. However, the second component of S1 coincides with the closure of the tricuspid valve. Hence, the two components are called as mitral component and tricuspid component. S2 also has two components, called aortic component and pulmonary component, which represent the closure of the aortic valve and pulmonary valve. Asynchronous closure of the aortic and pulmonary valves yields splitting of S2. The splitting has become widely recognized as a physiologic finding in both adults and children.

Studies about how to distinguish the aortic, pulmonary components and how to detect the splitting of S2 have progressed in recent years. The chirp model was introduced to represent aortic, pulmonary component, and separate them [3]. The time-frequency representation (TFR) method was used to analyze S2 to get the split [4]. It can be implemented by Short Time Fourier Transform (STFT). Considering S2 is a nonstationary signal, Wigner-Ville distribution (WVD) was adopted to analyze heart sounds [5]. To solve the interference terms of the WVD, the smoothed pseudo-Wigner Ville distribution (SPWVD) was used to obtain a better time-frequency resolution [5]. The SPWVD was improved by a reassignment method which can rearrange the coefficients of the SPWVD around new zero to yield a high resolution of TFR [6]. Combining Hilbert vibration decomposition (HVD) and SPWVD, Barma et al obtained quantitative measurement of the split [7-10]. It is referred to as HVD method in the following. In HVD method, an S2 signal is decomposed into several phase-fixed components and SPWVD operation is performed to each component to extract timing information. This paper proposes a framework to measure respiratory variations in the split of the second heart sound.

\section{Methods}

\subsection{Data collection}

Twelve young male subjects aged $24 \pm 1.8$ years participated in the experiments. All subjects provided their consents to participate in the experiments. They were asked to remain at rest for 10 min before data collection. Each subject was asked to lie on his back in a bed during data collection. Heart sounds, ECG lead II, and respiratory signals were simultaneously recorded at a sampling frequency of $2 \mathrm{kHz}$. A heart sound microphone sensor was placed at the left third intercostal space. The breathing transducer was a belt sensor positioned at the boundary of the thorax and abdomen to record respiratory movement. 
The time length for each recording has $150 \sim 180 \mathrm{~s}$.

\subsection{Investigation on S2 waveform changing modulated by respiration}

The respiratory phase signal is extracted from the respiratory signal, i.e., the respiratory signal is mapped to respiratory phase from $-\pi$ to $\pi$. Expiration begins at $-\pi$ and ends at 0 , meanwhile inspiration begins at 0 and ends at $\pi$. To achieve this, the respiratory signal and its Hilbert transform form the real part and imaginary part of an analytic signal. The respiratory phase is then obtained by the phase of the analytic signal.

To observe the changing visually, S2s are firstly segmented from the heart sound signal using the envelope method proposed by Liang et al [11]. The respiratory phases associated to the S2s are looked up from the respiratory phase signal with the reference of the S2s' timing. The S2s are re-sorted according to respiratory phase in ascending order. Then, the S2s are aligned in time domain and formed into a two dimensional matrix. The horizontal direction is in time domain and the vertical direction is in respiratory phase domain. These matrixes enable the split changes to be observed clearly. This preprocessing is repeated for the twelve subjects and the contour plot of these matrixes for one subject are shown in Fig. 1. A common phenomenon can be seen from the contour plot that the S2s have two parts. The first parts are aligned in time domain, whose waveforms have almost no change; however the timings of second parts are varying with respect to respiratory phase. That is to say, the splits between the two parts are modulated by the respiratory phase. The split decreases in expiration where the phase is from $-\pi$ to zero and the split increases in inspiration where the phase is from zero to $\pi$. The authors believe that the first parts are the aortic components and the second parts are the pulmonary components.

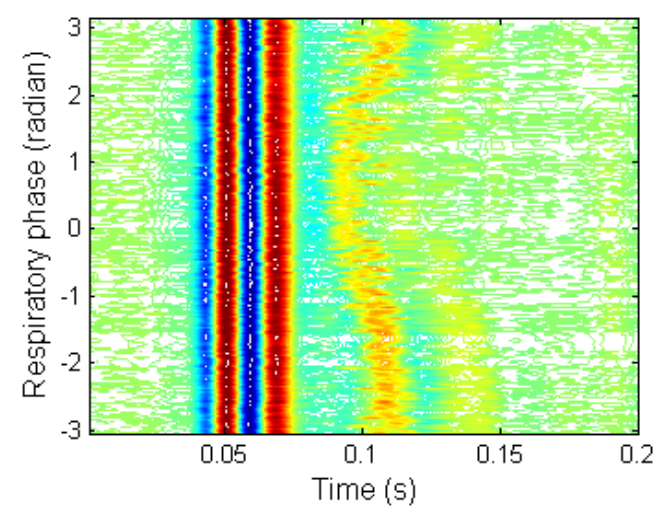

Figure 1. An example of second heart sounds demonstrated in joint time and respiratory phase domains

\subsection{System overview}

The overview of the system workflow has four steps. First, the heart sound signal and synchronous respiratory signal are bandpass filtered with zero-phase delay in the frequency band [5 200] Hz and [0.05 1] Hz, respectively. Second, segment all S2s from the heart sound signal and re-sort the S2s in joint time and respiratory phase domain. Third, discriminate aortic and pulmonary components. Fourth, measure the splits by weighted timing.

\subsection{Discrimination of aortic and pulmonary component}

It is assumed that the S2 is the sum of aortic and pulmonary components with the following model

$$
S_{2}(t, \theta)=a(t)+p(t-u(\theta))+v(t, \theta),
$$

where $a(t)$ is the aortic component, and $p(t-u(\theta))$ is the pulmonary component at the respiratory phase $\theta \cdot u(\theta)$ is the time delay modulated by respiration. $v(t, \theta)$ is random noise at respiratory phase $\theta$ to contaminate the second heart sound. The purpose of this article is to measure the respiratory split, $u(\theta)$. In detail, we have further assumptions based on above mentioned observation.

(1) $a(t)$ is assumed to have fixed waveform over the respiratory phase.

(2) $p(t-u(\theta))$ is the time shifted version of $p(t)$. It is a delayed pulmonary component caused by respiration.

(3) $v(t, \theta)$ is assumed to be zero mean both over time and over respiratory phase, i.e., $\int v(t, \theta) d t=0$ and $\int v(t, \theta) d \theta=0$. It may be colored, non-Gaussian and nonstationary.

An average operation over respiratory phase is implemented on the equation (1)

$\sum_{k=1}^{K} S_{2}\left(t, \theta_{k}\right) / K=\sum_{k=1}^{K}\left[a(t)+p\left(t-u\left(\theta_{k}\right)\right)+v\left(t, \theta_{k}\right)\right] / K$,

where $K$ is the number of S2s involved in operation. Based on the assumption that $v(t, \theta)$ is zero mean, the average below produces a zero vector.

$$
\sum_{k=1}^{K}\left[v\left(t, \theta_{k}\right)\right] / K=\mathbf{0}(t) .
$$

Therefore, the equation (2) becomes

$$
\sum_{k=1}^{K} S_{2}\left(t, \theta_{k}\right) / K=a(t)+\sum_{k=1}^{K}\left[p\left(t-u\left(\theta_{k}\right)\right)\right] / K .
$$

The average operation to the time shifted pulmonary components smooths the peaks and valleys. So, the average yields a small signal. To show the average operation clearly, an example is given in Fig. 2. The S2 signals (black lines) are over plotted. The solid bold red line is the averaged signal. It can be seen that the average of the time shifted pulmonary components produces so small signal that can be neglected considering the objective 
of this paper. Therefore, the second part in the right side of the equation (4) becomes

$$
\sum_{k=1}^{K}\left[p\left(t-u\left(\theta_{k}\right)\right)\right] / K \approx \mathbf{0}(t) .
$$

The estimated aortic component could be obtained by

$$
\hat{a}(t)=\sum_{k=1}^{K} S_{2}\left(t, \theta_{k}\right) / K \text {. }
$$

It means that the aortic component is discriminated by the ensemble average of S2s over respiratory phase. Furthermore, considering the equation (1), the pulmonary component could be discriminated by subtracting the aortic component from the $\mathrm{S} 2 \mathrm{~s}$,

$$
\hat{p}(t-u(\theta))=S_{2}(t, \theta)-\hat{a}(t) .
$$

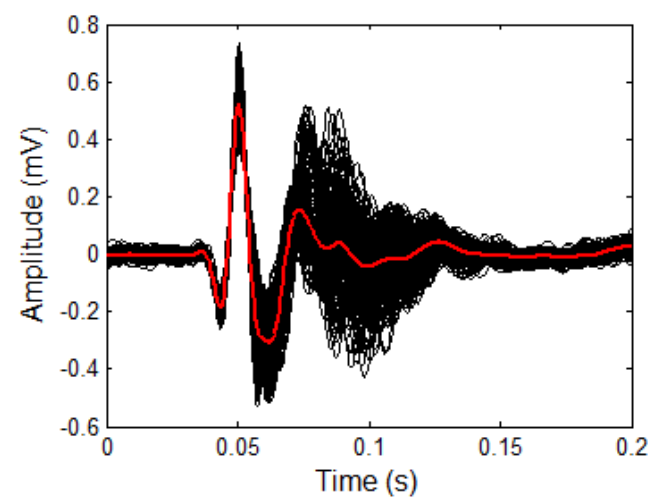

Figure 2. Average operation on S2s over respiratory phase. Black lines are the S2s and the solid red line is the average.

\subsection{Estimation of time indices}

The timings of the estimated aortic and pulmonary component are extracted from envelope peak position. It is operated in the following. Firstly, get the upper envelope of the absolute aortic component using the local maxima with the help of cubic spline interpolation. The way to calculate the upper envelope is like extracting the upper envelope in empirical mode decomposition (EMD) [12]. Then, the local peaks' amplitude and associated peaks' location of the powered envelope are searched

$$
\left[\boldsymbol{A}^{a}, \boldsymbol{T}^{a}\right]=\text { findpeaks }\left(\left[\operatorname{en}_{a}(t)\right]^{k}\right),
$$

where $e n_{a}(t)$ is the upper envelope of aortic component and $k$ is empirically set as 8 . The function of this power operation is to make the dominant peaks protruding. $\boldsymbol{A}^{a}=\left[A_{1}^{a}, A_{2}^{a}, \cdots, A_{M}^{a}\right]$ is a vector consisting of peak amplitude, $\boldsymbol{T}^{a}=\left[T_{1}^{a}, T_{2}^{a}, \cdots, T_{M}^{a}\right]$ is a vector consisting of peak position, and $M$ is the number of peaks. So, the timing of the aortic component, $t_{a}$, is defined as weighted peak position

$$
t_{a}=\left(\sum_{m=1}^{M} W_{m}^{a} T_{m}^{a}\right) / M
$$

where $W_{m}^{a}$ is weighted coefficient. Similarly, get the upper envelope of the absolute pulmonary component using the local maxima with the help of cubic spline interpolation.

The peaks of the estimated pulmonary component can be determined by

$$
\left[\boldsymbol{A}^{p}, \boldsymbol{T}^{p}\right]=\text { findpeaks }\left(\left[e n_{p}(t)\right]^{k}\right),
$$

where $e n_{p}(t)$ is the upper envelope of pulmonary component and $k$ is empirically set as 8 . $\boldsymbol{A}^{p}=\left[A_{1}^{p}, A_{2}^{p}, \cdots, A_{N}^{p}\right]$ is a vector consisting of peak amplitude, $\boldsymbol{T}^{p}=\left[T_{1}^{p}, T_{2}^{p}, \cdots, T_{N}^{p}\right]$ is a vector consisting of peak position, and $N$ is the number of peaks. So, the timing of the pulmonary component, $t_{p}$, is defined as weighted peak positon

$$
t_{p}=\left(\sum_{n=1}^{N} W_{n}^{p} T_{n}^{p}\right) / N,
$$

where $W_{n}^{p}$ is weighted coefficient. The split between the aortic and pulmonary component is then estimated by the timing difference

$$
\hat{u}(\theta)=\left|t_{a}-t_{p}\right| \cdot
$$

\section{Experiments}

The authors have collected data from 12 subjects. Each recording has several hundred cardiac cycles. A pair of respiratory phase and split can be obtained from each cardiac cycle based on the proposed method, which can be represented by a star in the joined domain. Hence, the respiratory variations in splitting of the second heart sound can be clearly illustrated by the stars where the measured splits are re-sorted in respiratory phase in ascending order, seen in Fig. 3. It can be seen that the split gradually increases with the phase increasing from 0 to $\pi$ (inspiration) or decreases with the phase from $-\pi$ to 0 (expiration). The minimum split generally occurs at phase zero and the split value is about $20 \mathrm{~ms}$. Meanwhile, the maximum split is about $50 \mathrm{~ms}$ at phase $\pi$ or $-\pi$. These values are comparable to the previous physiological results. The experimental results imply that the proposed method is reasonable to monitor the respiratory variations in the split of the second heart sound. Furth more, each step of the proposed method can run automatically without any human intervention.

\section{Conclusions}

The second heart sound consists of aortic and pulmonary components. Previous physiological studies disclosed that the time delay between the two components is modulated by respiration. A novel method is proposed to quantitatively measure the delay using the respiratory modulation. A model is set up to assume that the second heart sound is the sum of aortic component and delayed 
pulmonary component. The aortic component could be estimated by the average of the second heart sounds over respiratory phase. Subsequently, the pulmonary component could be estimated by subtracting. The timing of the two components is further determined by the weighted powered envelope peak position. The proposed method is evaluated by practical data collected from healthy subjects. The experimental results clearly show that the detected splits of the second heart sound vary with respiratory phase. The values of the split and the trend of the splits coincide with previous results very well. The results suggest the proposed method could be useful in non-invasively monitoring the hemodynamic response to respiration.
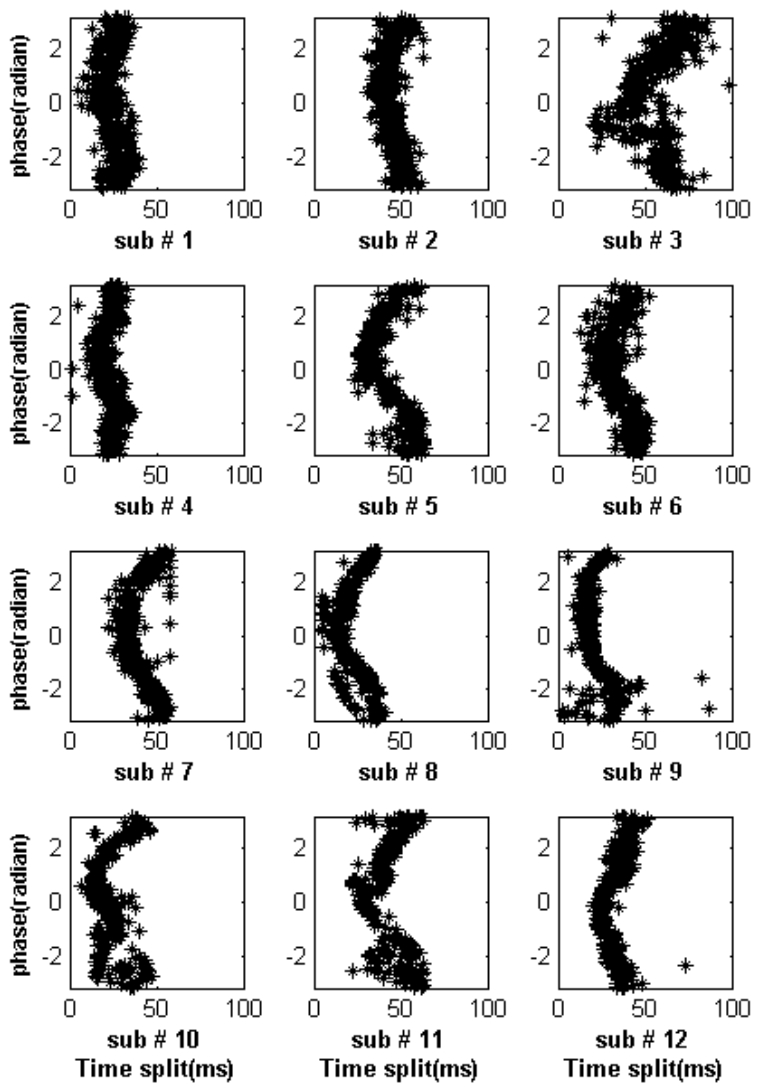

Figure 3. Measured respiratory splits for the 12 subjects by the proposed method

\section{Acknowledgements}

This work was supported in part by the National Natural Science Foundation of China under Grant No. 61471081, No. 61601081 and Fundamental Research Funds for the Central Universities under Grant No. DUT15QY60, DUT16QY13, DC201501056, and DCPY2016008.

\section{References}

[1] A A Luisada, C K Liu, C Aravanis, M Testelli, J. Morris, B A Chicago. On the mechanism of production of the heart sounds. American Heart Journal, 1958, 55, 383-399.

[2] T E Piemme, G O Barnett, L Dexter. Relationship of heart sounds to acceleration of blood flow. Circulation Research, 1966, 18, 303-315.

[3] J Xu, L-G Durand, P Pibarot. Extraction of the aortic and pulmonary components of the second heart sound using a nonlinear transient chirp signal model. IEEE Transactions on Biomedical Engineering, 2001, 48, 277-283.

[4] T S Leung, P R White, J Cook, W B Collis, E Brown, A P Salmon. Analysis of the second heart sound for diagnosis of paediatric heart disease. IEE Proceeding -Sci. Meas. Technolo., 1998, 145, 285-290.

[5] A Djebbari, F Reguig. Smoothed pseudo wigner-ville distribution of normal and aortic stenosis heart sounds. Journal of Mechnamics in Medicine and Biology, 2005, 5, 415-428.

[6] A Djebbari, F Bereksi-Reguig. Detection of the valvar split within the second heart sound using the reassigned smoothed pseudo Wigner-Ville distribution. Biomedical Engineering Online, 2013, 12, 37.

[7] S Barma, B Chen, K L Man, J Wang. Quantitative measurement of split of the second heart sound. IEEE Transactions on computational biology and bioinformatics, 2015, 12, 851-860.

[8] M Feldman. Hilbert transform application in mechanical vibration. John Wiley Sons, Ltd., Publication, 2011.

[9] F Augen, P Flandrin, Y Lin, S Mclaughlin, S Meignen, T Oberlin, $\mathrm{H}$ Wu. Time-frequency reassignment and synchrosqueezing. IEEE signal processing magazine, 2013, 30, 32-41.

[10] F Auger, P Flandrin. Improving the readability of timefrequency and time-scale representations by the reassignment method. IEEE Transactions on signal processing, 1995, 43, 1068-1089.

[11] H Liang, S Lukkarimen, I Hartimo. Heart sound segmentation algorithm based on heart sound envelogram. Computer in Cardiology, 1997, 24, 105-108.

[12] N E Huang, Z Shen, S R Long, M C Wu, H H Shih, Q Zheng, $\mathrm{N}$ C Yen, C C Tung, H H Liu. The empirical mode decomposition and the Hilbert spectrum for nonlinear and non-stationary time series analysis. Proceedings of the Royal Society A-Mathematical Physical and Engineering Sciences, 1971, 454, 903-995.

Address for correspondence.

Name. Hong Tang

Full postal address. Department of Biomedical Engineering, Dalian University of Technology, Dalian 116024, China

E-mail address (optional). tanghong@dlut.edu.cn 